\title{
Exotic shapes in ${ }^{40} \mathrm{Ca}$ and ${ }^{36} \mathrm{Ar}$ studied with Antisymmetrized Molecular Dynamics
}

\author{
Y. Kanada-En'yo, M. Kimura* and H. Horiuchi** \\ Institute of Particle and Nuclear Studies, High Energy Accelerator Research Organization, \\ Tsukuba 305-0801, Japan \\ The Institute of Physical and Chemical Research, Wako 351-0198, Japan \\ * Department of Physics, Kyoto University, Kyoto 606-01, Japan
}

\begin{abstract}
The structures of the ground and excited states of $s d$-shell nuclei: ${ }^{40} \mathrm{Ca}$ and ${ }^{36} \mathrm{Ar}$ were studied with a method of antisymmetrized molecular dynamics. Recently observed rotational bands were described with deformed intrinsic states which are dominated by excited configurations such as $4 p-4 h, 8 p-8 h$ and $4 p-8 h$ states. The results showed the coexistence of various kinds of exotic shapes with oblate, prolate and large prolate deformatios in the lowenergy region of ${ }^{40} \mathrm{Ca}$. Possible cluster aspects in heavy $s d$-shell nuclei were implied in these exotic shapes.
\end{abstract}

\section{INTRODUCTION}

Owing to the experiments of gamma-ray measurements, many excited bands in the nuclei near ${ }^{40} \mathrm{Ca}$ have been recently observed [1,2]. The rotational bands with large moments of inertia are hot subjects relating with super deformations in $s d$-shell nuclei. In ${ }^{40} \mathrm{Ca}$, there exist many low-lying bands, which imply the coexistence of various shapes. It is interesting problem why the shape coexistence occurs even in the doubly magic nucleus. The shape coexistence problem and the mechanism of deformations in these $s d$-shell nuclei is one of the attractive subjects.

Our interests are possible exotic shapes and cluster aspects in the deformed excited states. It has been already known that clustering is one of the essential features in light nuclei . Exotic shapes are formed due to cluster structures in some light nuclei, For example, a famous cluster structure in ${ }^{12} \mathrm{C}$ is three $\alpha$ clusters which form a triangle shape. Parity doublets of the rotational bands in ${ }^{20} \mathrm{Ne}$ are described by a parity asymmetry shape with a ${ }^{16} \mathrm{O}+\alpha$ cluster structure. It is a long problem whether or not cluster aspects appear in heavier nuclei. Many of the familier cluster structures are those in light stable nuclei. In the recent experimental and theoretical research [3 7], cluster structures were suggested in light unstable nuclei such as ${ }^{10} \mathrm{Be}$ and ${ }^{12} \mathrm{Be}$, where new type clusters such as ${ }^{6} \mathrm{He}$ and ${ }^{8} \mathrm{He}$ are proposed. Our aim in this paper is systematic study of the $s d$-shell nuclei near ${ }^{40} \mathrm{Ca}$ to find possible exotic states with cluster aspects. 
For the systematic study of ground and excited states of $s d$-shell nuclei, theoretical difficulties exist in the coexistence of cluster and mean-field aspects. Needless to say, cluster aspects are important in light nuclei, while the mean-field is an essential feature in heavy nuclei. Since the $s d$-shell is an inter-mediate mass-number region, where both aspects should be significant, we need a miscroscopic method beyond the traditional clsuter models. Namely, we adopt a method of antisymmetrized molecular dynamics(AMD) 8 11,5]. The method AMD was applied for light nuclei and has been proved to be a powerful approach to describe the ground and excited states of unstable nuclei as well as stable nuclei. In the pioneering study with the AMD method, the structure changes on the ylast line of ${ }^{20} \mathrm{Ne}$ are explained in terms of alteration of the cluster structure [9]. In the recent studies of light unstable nuclei with AMD, a new cluster aspect in unstable nuclei was suggested that cluster cores develop in the deformed neutron mean-field in neutron-rich nuclei.

In the present work, we adopt a new effective force with a finite-rage three-body term, with which the binding energies and the radii of ${ }^{4} \mathrm{He},{ }^{16} \mathrm{O}$ and ${ }^{40} \mathrm{Ca}$ are systematically reproduced, because the usual effective forces used in the AMD study, such as Minnesota forces, Volkov forces and Modified Volkov forces, have serious problems in reproducing these basic properties in the wide mass-number region.

In this paper, the structures of the ground and excited states of ${ }^{40} \mathrm{Ca}$ and ${ }^{36} \mathrm{Ar}$ are studied by the AMD method. We investigate the shape coexistence problem of these nuclei. The mechanism of deformations are discussed, focusing on cluster aspects. We also introduce the AMD study of neuton-rich $s d$-shell nuclei near ${ }^{32} \mathrm{Mg}$.

\section{FORMULATION}

In this section, the formulation of AMD for the nuclear structure study of ground and excited states is briefly explained. For more detailed descriptions of the AMD framework, the reader is referred to Refs [9 11].

The wave function of a system is written by AMD wave functions,

$$
\Phi=c \Phi_{A M D}+c^{\prime} \Phi_{A M D}^{\prime}+\cdots
$$

An AMD wave function of a nucleus with a mass number $A$ is a Slater determinant of Gaussian wave packets;

$$
\begin{gathered}
\Phi_{A M D}(\mathbf{Z})=\frac{1}{\sqrt{A !}} \mathcal{A}\left\{\varphi_{1}, \varphi_{2}, \cdots, \varphi_{A}\right\}, \\
\varphi_{i}=\phi_{\mathbf{X}_{i}} \chi_{\xi_{i}} \tau_{i}:\left\{\begin{array}{l}
\phi_{\mathbf{X}_{i}}\left(\mathbf{r}_{j}\right) \propto \exp \left[-\nu\left(\mathbf{r}_{j}-\frac{\mathbf{X}_{i}}{\sqrt{\nu}}\right)^{2}\right], \\
\chi_{\xi_{i}}=\left(\begin{array}{c}
\frac{1}{2}+\xi_{i} \\
\frac{1}{2}-\xi_{i}
\end{array}\right),
\end{array}\right.
\end{gathered}
$$

where the $i$ th single-particle wave function $\varphi_{i}$ is a product of the spatial wave function $\phi_{\mathrm{X}_{i}}$, the intrinsic spin function $\chi_{\xi_{i}}$ and the iso-spin function $\tau_{i}$. The spatial part $\phi_{\mathrm{X}_{i}}$ is presented by variational complex parameters $X_{1 i}, X_{2 i}, X_{3 i} \cdot \chi_{\xi_{i}}$ is the intrinsic spin function defined by $\xi_{i}$, and $\tau_{i}$ is the iso-spin function which is fixed to be up(proton) or down(neutron) in 
the present calculations. The values $\mathbf{Z} \equiv\left\{X_{n i}, \xi_{i}\right\}(n=1,2,3$ and $i=1, \cdots, A)$ are the variational parameters which express an AMD wave function.

In order to obtain the wave functions of ground and excited states, we perform a generator coordinate method in the framework of AMD. First we vary the energy of the parity eigen state projected from an AMD wave function under a constraint that the total oscillator quanta must equal to a given number $\mathcal{N}$ as $\left\langle a a^{\dagger}\right\rangle=\mathcal{N}$. The energy variation is numerically calculated by a frictional cooling method [9]. An energy curve is obtained as a function of the coordinate $\mathcal{N}$ in the constraint. In the second step, the spinparity eigen states projected from the obtained AMD wave functions are superposed by diagonalizing Hamiltonian and Norm matrices, $\left\langle P_{M K^{\prime}}^{J \pm} \Phi_{A M D}\left(\mathbf{Z}_{i}\right)|H| P_{M K^{\prime \prime}}^{J \pm} \Phi_{A M D}\left(\mathbf{Z}_{j}\right)\right\rangle$ and $\left\langle P_{M K^{\prime}}^{J \pm} \Phi_{A M D}\left(\mathbf{Z}_{i}\right) \mid P_{M K^{\prime \prime}}^{J \pm} \Phi_{A M D}\left(\mathbf{Z}_{j}\right)\right\rangle$, We call the present calculations as $\operatorname{VBP}($ variation before projection) because the variation is performed before the spin projection.

\section{INTERACTIONS}

The ordinary effective forces with no three-body term such as the Volkov force or with a zero-range three-body term like the MV1 force are not appropriate to describe the binding energies and radii of nuclei covering wide mass number region from $\alpha$ to ${ }^{40} \mathrm{Ca}$. Hence we use an interaction containing a finite-range three-body term in the present calculations. The central part of the interaction is explained by a conbination of the two-body and three-body terms. The interaction parameters used in the present paper are as follows,

$$
\begin{gathered}
V_{\text {central }}=\sum_{i<j} V^{(2)}+\sum_{i<j<k} V^{(3)}, \\
V^{(2)}=\left(1-m+b P_{\sigma}-h P_{\tau}-m P_{\sigma} P_{\tau}\right)\left\{V_{a} \exp \left[-\left(\frac{r_{12}}{r_{a}}\right)^{2}\right] V_{b} \exp \left[-\left(\frac{r_{12}}{r_{b}}\right)^{2}\right]\right\} \\
+V_{c} \exp \left[-\left(\frac{r_{12}}{r_{c}}\right)^{2}\right], \\
V^{(3)}=V_{d} \exp \left[-d\left(r_{12}^{2}+r_{23}^{2}+r_{31}^{2}\right)^{2}\right], \\
r_{a}=1.2 \mathrm{fm}, r_{b}=0.7 \mathrm{fm}, r_{c}=0.9 \mathrm{fm}, V_{d}=600 \mathrm{MeV}, d=0.8 \mathrm{fm}^{-2} \\
m=0.193, b=-0.185, h=0.37,
\end{gathered}
$$

where the width and strength parameters are chosen so as to reproduce reasonably the sizes of $\alpha$ and ${ }^{40} \mathrm{Ca}$, and the binding energies of $\alpha,{ }^{16} \mathrm{O}$ and ${ }^{40} \mathrm{Ca}$. In choosing parameters, $\alpha+\alpha$ phase shifts and the saturation property of symmetric nuclear matter have been also taken into consideration. The adopted interaction is a sum of the central force, the G3RS spin-orbit force [12 with the strength $u_{l s}=2500 \mathrm{MeV}$ and the Coulomb force.

\section{RESULTS}

The excited deformed bands in the ${ }^{40} \mathrm{Ca}$ and ${ }^{36} \mathrm{Ar}$ have been recently observed in the gamma-ray measurements [1,2], which reveal the existence of many low-lying bands in these

nuclei. In the experimental level scheme of ${ }^{40} \mathrm{Ca}$, the rotational band with a large moment of inertia (No.1 in Fig.11) is a hot topic relating with a possible super deformation. 
FIG. 1. The experimental data of the energy levels of ${ }^{40} \mathrm{Ca}$. The figure is taken from [i]].

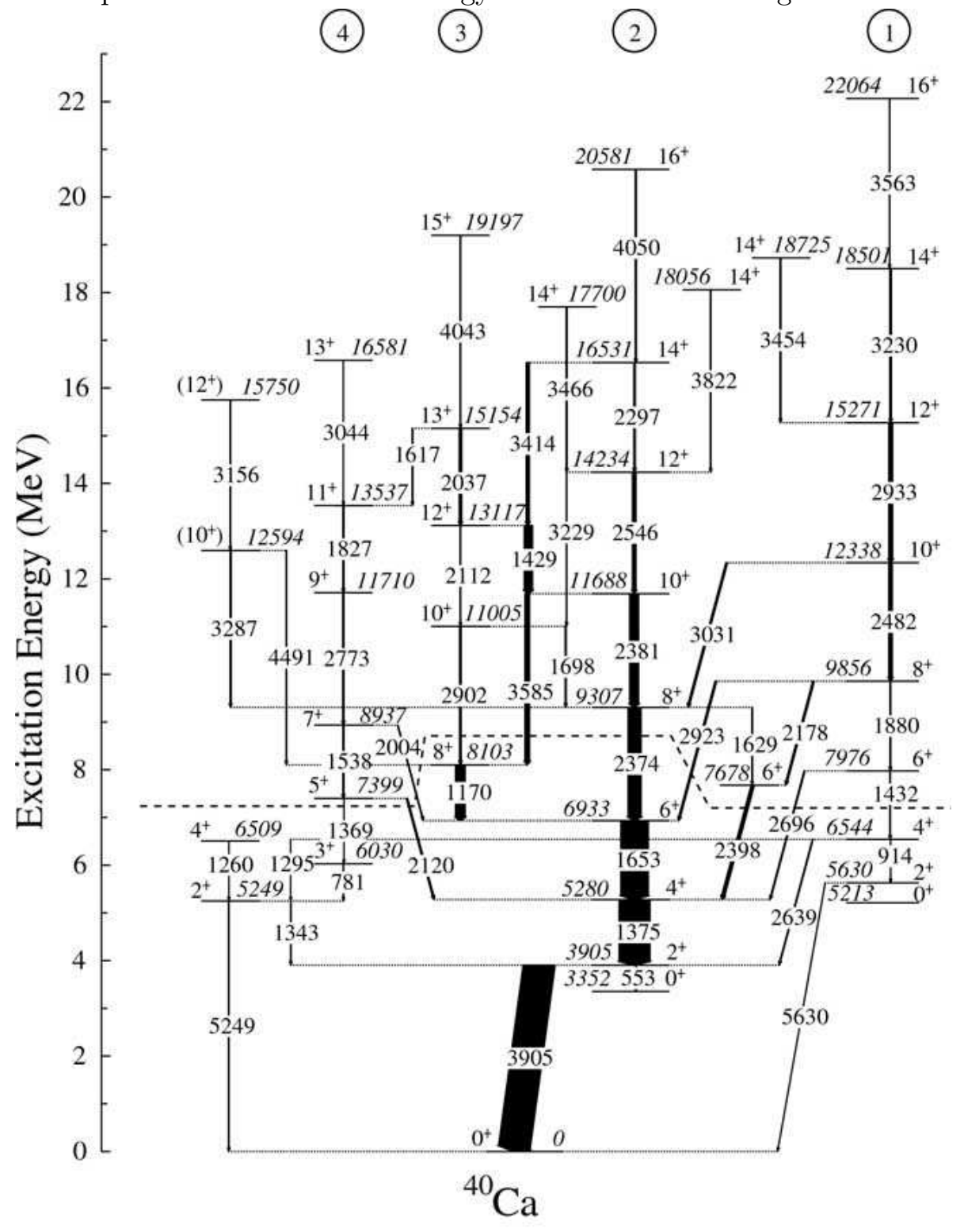


FIG. 2. The theoretical energy levels of ${ }^{40} \mathrm{Ca}$ obtained after the diagonalization with respect to the spin-parity projected states.

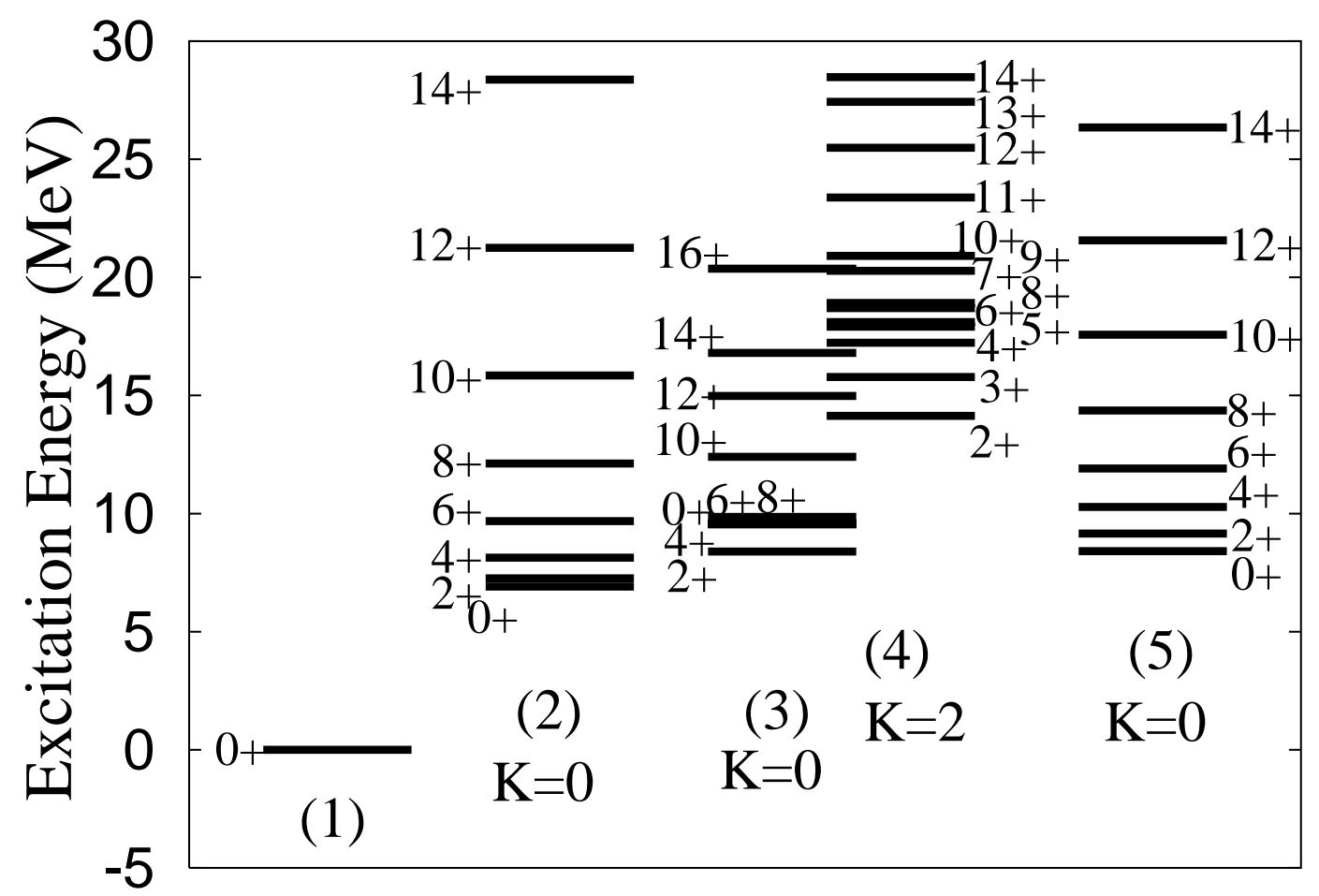

We study the ground and excited states of ${ }^{40} \mathrm{Ca}$ and ${ }^{36} \mathrm{Ar}$, with the method of a generator coordinate method in the framework of AMD with respect to the total oscillator quanta. In the energy curves obtained after spin-projection as a function of the oscillator quanta, we can not necessarily find local minima except for the absolute minimum. However, after diagonalization of the Hamiltonian and Norm matrices, the energy curves for the excited states have other local minima due to the conditions orthogonal to the lower states. As a result, we obtain many low-liying rotational bands (Fig.2), each of which is dominated by the spin-parity projected states from an intrinsic AMD wave function.

By analyzing deformations of these dominant intrinsic states, it is found that various shapes coexist in ${ }^{40} \mathrm{Ca}$. The interesting point is that each of the intrinsic states in ${ }^{40} \mathrm{Ca}$ is well described by either of $0 p-0 h, 4 p-4 h$ or $8 p-8 h$ configurations. First we obtain the normal spherical ground state with the doubly closed $s d$-shell configuration. With the increase of the oscillator quanta, the deformed excited states appear in the low-energy region. In the region concerning $4 p-4 h$ states, there exist opposite shapes as two local minimum states. One is an oblate shape with the deformation parameter $\beta=0.2$, while the other is a prolate deformation with $\beta=0.2$. The oblate state provides the excited $K^{\pi}=0^{+}$band (2), and the bands (3) and (4) with $K^{\pi}=0^{+}, 2^{+}$are dominated by the states projected from the prolate intrinsic state. The reason for the higher $0^{+}$state than the $2^{+}$state in the $K^{\pi}=0^{+}$band (3) is the mixing with the ground state. With a larger oscillator quanta, we find another largely prolate deformation which is dominated by $8 p-8 h$ configurations. The energy levels of the band (5) obtained from this large deformation indicate a large moment 
TABLE I. The theoretical values (W.u.) of $E 2$ transition strength in the excited $K^{\pi}=0^{+}$ bands.

\begin{tabular}{lccc}
\hline \hline transition & band $(2)$ & band(3) & band(5) \\
\hline $2^{+} \rightarrow 0^{+}$ & 11 & 2 & 30 \\
$4^{+} \rightarrow 2^{+}$ & 16 & 21 & 53 \\
$6^{+} \rightarrow 4^{+}$ & 16 & 13 & 61 \\
$8^{+} \rightarrow 6^{+}$ & 17 & 14 & 78 \\
\hline \hline
\end{tabular}

of inertia of this rotational band. It is very surprising that the excited band with such highly excited configurations as $8 p-8 h$ exists in low-energy region. We identify this band as the experimentally observed super-deformed band which starts from the $0^{+}$state at $5.2 \mathrm{MeV}$.

As shown in Table \&, the theoretical values of the $E 2$ transition strength in the band (5) are extremely large comparing with those in other excited band. According to a quantitative comparison of the strength in the band (5) with the experimental data, the theoretical E2 strength for $4^{+} \rightarrow 2^{+}$is smaller than the corresponding experimental data $B(E 2 ; 6.54 \mathrm{MeV} \rightarrow 5.63 \mathrm{MeV})=100(W . u$.$) . Therefore, it is conjectured that the intrinsic$ deformation $\beta=0.4$ of the band (5) in the present results must be still small compared with the value $\beta=0.6$ for a typical super deformation. The underestimation of the deformations in the present theory will be improved by the superposition along generator coordinates and also the extension of the single-particle wave functions.

As mentioned above, the point in the present results of ${ }^{40} \mathrm{Ca}$ is the coexistence of various shapes: the spherical state, the oblate shape, the normal prolate deformation and the large prolate deformation. By analysing the intrinsic structures of these deformed states, exotic shapes and cluster aspects are revealed. The density distributions of the dominant intrinsic states of the excited bands are presented in Fig. 3. As shown in Fig. 3 , the oblate state (2) has a hexagon structure like a three-leaf clover which consists of three ${ }^{12} \mathrm{C}$ cores surrounding an $\alpha$ at the center. In the super deformation, the system has a parity-asymmetry shape like a pear because of a ${ }^{28} \mathrm{Si}+{ }^{12} \mathrm{C}$-like clustering. We focus the characteristics of the ${ }^{28} \mathrm{Si}+{ }^{12} \mathrm{C}$-like cluster structures in the super deformation. The first point is that the clusters are not weak coupling but are strong coupling ones. It is interesting that the ${ }^{12} \mathrm{C}$ cluster consists of four nucleons in the $s d$-shell and eight nucleons in the $p f$-shell. Namely, it is a inter-shell cluster which lies over different shells. Because of the strong coupling feature, it is expected that the deformed mean-field effect can be important as well as cluster aspects in the state. In second, if such a cluster structure indeed develops, it forms a parity asymmetric deformation, which may provide the parity doublets made of positive and negative-parity bands. Thirdly, it is reasonable that both clusters are the sub-shell closed nuclei. In other words, the stability of these cluster cores is based on the shell effects of the sub-shell closure. For further analysis of the cluster aspects, we need advanced calculations where the residual effects such as the coupling of other cluster channels, the tail of inter-cluster motion, and the core deformations are more carefully taken into consideration.

It should be pointed that the present results imply the possible exotic shapes due to the cluster effects such as ${ }^{12} \mathrm{C}$ cores, though there are few experimental evidences of the 
FIG. 3. The density distributions of the intrinsic states before spin-parity projections. The notations (2),(3),(5) are same as those in Fig.2. In each figure, the density of the dominant AMD wave function of each band is presented. The matter density is integrated along the axis with the largest moment of inertia.
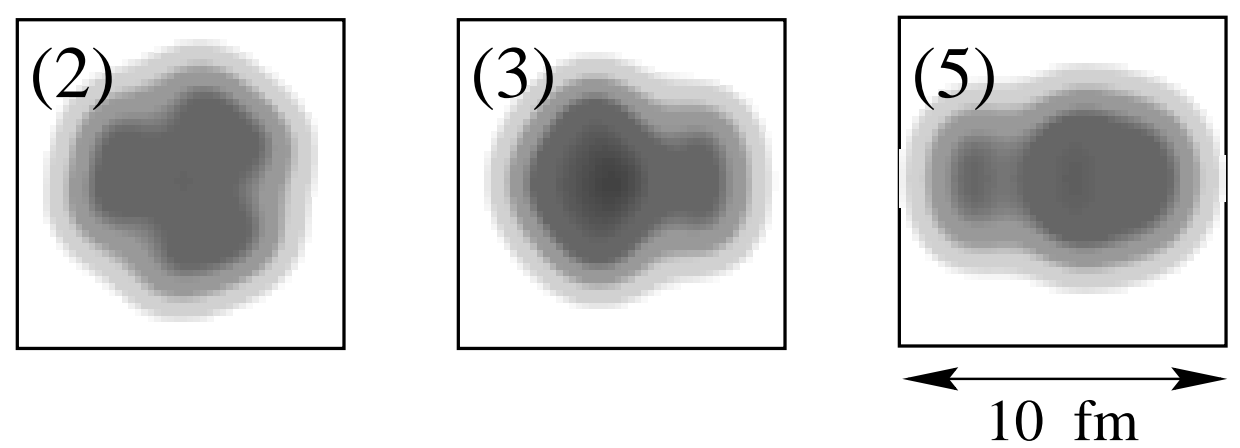

existence of clusters in ${ }^{40} \mathrm{Ca}$. The investigation of the level structures of the negativeparity states is one of the probes, because the parity-doublets of positive and negative-parity bands support a cluster structure as the origin of a parity-asymmetry intrinsic state. The present calculations predict a $K^{\pi}=0^{-}$band with the band-head $1^{-}$state at $11.7 \mathrm{MeV}$, which is about $3 \mathrm{MeV}$ higher than theoretical band-head energy of the positive-parity super deformation. The deformation of the dominant intrinsic state is rather large as $\beta=0.3$ and the intrinsic structure is similar to that of the super deformation in the positive-parity states. Therefore we consider this negative-parity band as a candidate of the parity doublet. Strictly speaking, in negative parity states, the rotational levels fragments because the state mixing between bands is rather strong. As a result, the intra-band $E 2$ transitions in the $K^{\pi}=0^{-}$ band are not so strong as those in the positive-parity band of the super deformation.

In the theoretical results of ${ }^{36} \mathrm{Ar}$, we find excited bands with a large prolate deformation $\beta=0.3$ which are dominated by $4 p-8 h$ configurations. This band starts from a $4 \mathrm{MeV}$ excitation energy, and well corresponds to the newly observed rotational band with a large moment of inertia in the experimental data [2].

At the end of this section, I briefly report the AMD study on neutron-rich $s d$-shell nuclei near ${ }^{32} \mathrm{Mg}$ calculated by one of the authors(M. K.). The structures of the ground and excited states of ${ }^{32} \mathrm{Mg}$ are hot subjects concerning the island of inversion of a neutron magic number $N=20$. The ground and excited states of $\mathrm{Mg}$ isotopes are calculated by an extended version of AMD with the Gogny force. The calculations well reproduce the energy levels of ${ }^{32} \mathrm{Me}$ and the features of the island of inversion. In the neutron-rich $s d$-shell nuclei, the deformed neutron mean-field plays an important role. The unique predictions in this work are the low-lying negative parity states in ${ }^{32} \mathrm{Mg}$. Since the nuclei ${ }^{32} \mathrm{Mg}$ and ${ }^{40} \mathrm{Ca}$ have the same neutron number, it is interesting future problem to compare the intruder states of ${ }^{32} \mathrm{Mg}$ with the excited states of ${ }^{40} \mathrm{Ca}$.

\section{SUMMARY}

The structures of the ground and excited states of $s d$-shell nuclei: ${ }^{40} \mathrm{Ca}$ and ${ }^{36} \mathrm{Ar}$ were studied with the method of antisymmetrized molecular dynamics. The recently observed 
excited bands were well described with the deformed intrinsic states in the present calculations. It was found that various kinds of shape coexist in the low-energy region. In ${ }^{40} \mathrm{Ca}$, it was suggested in the present results that the oblate shape, the normal prolate deformation and the large prolate deformation coexist in the low-lying excited bands besides the spherical ground state. The oblate, normal prolate, and the large prolate states are explained by the excited configurations with $4 p-4 h, 4 p-4 h$, and $8 p-8 h$, respectively. It is a mystery that the rotational band with the highly excited configurations $(8 p-8 h)$ starts from the low-energy region. The exotic shapes due to the cluster effects were implied in the intrinsic structures of these deformed states. The present results suggested possible cluster aspects in heavy $s d$-shell nuclei.

\section{ACKNOWLEDGMENTS}

Authors would like to thank Professors A. Tohsaki, Y. Akaishi and K. Ikeda for helpful discussions and comments. They are also thankful to Dr. N. Itagaki and E. Ideguchi for many discussions. This work was partially performed in the "Research Project for Study of Unstable Nuclei from Nuclear Cluster Aspects" sponsored by Institute of Physical and Chemical Research (RIKEN). The computational calculations of this work are supported by the Supercomputer Project No.58, No.70 of High Energy Accelerator Research Organization(KEK), and Research Center for Nuclear Physics(RCNP) in Osaka University. 


\section{REFERENCES}

[1] E. Ideguchi et al., Phys. Rev. Lett. 87, 222501(2001)

[2] C.E.Svensson et al., Phys. Rev. Lett. 85, 2693(2000)

[3] M. Freer, et al., Phys. Rev. Lett. 82, 1383 (1999); M. Freer, et al., Phys. Rev. C 63, 034301 (2001).

[4] A. Saito, et al., Proc. Int. Sympo. on Clustering Aspects of Quantum Many-Body Systems, eds A. Ohnishi, N. Itagaki, Y. Kanada-En'yo and K. Kato, (World Scientific Publishing Co.) (to be published).

[5] Y. Kanada-En'yo, H. Horiuchi and A. Doté, Phys. Rev. C 60, 064304(1999).

[6] N. Itagaki and S. Okabe, Phys. Rev. C 61, 044306 (2000);

[7] Y. Kanada-En'yo, Phys. Rev. C 66, 011303 (2002)

[8] A. Ono, H. Horiuchi, T. Maruyama, and A. Ohnishi, Prog. Theor. Phys. 87, 1185 (1992).

[9] Y. Kanada-En'yo and H. Horiuchi, Prog. Theor. Phys. 93, 115 (1995).

[10] Y. Kanada-En'yo, A. Ono, and H. Horiuchi, Phys. Rev. C 52, 628 (1995); Y. KanadaEn'yo and H. Horiuchi, Phys. Rev. C 52, 647 (1995).

[11] Y. Kanada-En'yo, Phys. Rev. Lett. 81, 5291 (1998).

[12] N. Yamaguchi, T. Kasahara, S. Nagata, and Y. Akaishi, Prog. Theor. Phys. 62, 1018 (1979); R. Tamagaki, Prog. Theor. Phys. 39, 91 (1968). 